\title{
Utilisation d'un mini-pressiomètre pour la mesure directe du frottement à l'interface sol pulvérulent-inclusion
}

\author{
The use of a minipressuremeter for a direct measure of friction \\ between an inclusion and a cohesionless soil
}

\author{
A. ABDERRAHIM, J.P. TISOT \\ Laboratoire de Géomécanique, ENSG Nancy \\ CRNS-GRECO Géomatériaux*
}

Rev. Franç. Géotech. n 61, pp. 63-74 (décembre 1992)

\section{Résumé}

Le comportement d'une inclusion dans un sol et plus particulièrement la connaissance du frottement mobilisé à l'interface joue un rôle fondamental dans les problèmes de renforcement des sols. La détermination du coefficient de frottement a été réalisée suivant différentes approches, tant au laboratoire qu'in situ. Une nouvelle approche basée sur I'utilisation d'un mini-pressiomètre durant un essai d'arrachement est proposée. L'avantage de ce dispositif réside dans le fait suivant : la contrainte normale à l'interface peut être contrôlée durant l'essai ce qui permet le choix de deux procédures d'essais :

- arrachement à volume constant avec mesure de la variation de la contrainte normale ;

- arrachement à contrainte normale constante avec mesure de la variation de volume.

Ces arrachements sont réalisés soit d'une manière continue, soit par paliers.

Cet article décrit les techniques mises en œuvre pour la réalisation de l'essai et donne les premiers résultats obtenus suivant différentes procédures expérimentales.

\begin{abstract}
The behaviour of an inclusion in a soil and particularly the knowledge of the friction value is of paramount importance for the soil reinforcement problems. The determination of the friction coefficient has been realised with differents approachs : in laboratory and in situ. In this paper, a new approach based on the use of a minipressuremeter during the wrenching is proposed. The advantage of this apparatus lies in the following fact : the normal stress at the interface can be controlled during the test, which allows the choice of two test procedures : the normal stress is kept constant during shearing and the variation of volume is measured; the volume is kept constant and the variation of the normal stress is measured. A progressif or static wrenching are realised. The procedure and the first results obtained along different experimental conditions are presented in this paper.
\end{abstract}

- Rue du Doyen Marcel-Roubault, BP 40, 54501 Vandœuvre-lès-Nancy. 


\section{INTRODUCTION}

L'amélioration du sol est une préoccupation importante des mécaniciens du sol. Cette amélioration se fait entre autre par des procédés mécaniques comme le compactage, le drainage ou encore l'inclusion d'éléments résistants. Le renforcement par des éléments résistants (pieux, ancrages, etc.) peut être apprécié par la connaissance du comportement local du matériau composite sol-inclusion. Le phénomène principal qui régit ce « comportement local » est le frottement.

L'approche expérimentale de ce comportement, au laboratoire ou in situ, paraît indispensable pour une compréhension plus objective tenant compte des problèmes réels rencontrés sur le terrain.

Dans cette perspective, ce travail est orienté principalement vers l'étude du comportement à l'interface sol pulvérulent-inclusion à travers une nouvelle approche permettant de mesurer directement le frottement mobilisé à l'interface à l'aide d'un mini-pressiomètre, mis en place dans le sol, puis arraché suivant une méthodologie proche de celle définie par PHILIPPONNAT G. pour le phicomètre (1986).

La connaissance de la contrainte normale à l'interface et de son évolution lors d'un cisaillement sont des paramètres importants dans la compréhension des phénomènes de mobilisation du frottement. Ils sont fonction essentiellement de l'état initial, de la compressibilité des sols, du volume de la zone intéressée par le cisaillement mais ces facteurs sont difficiles à apprécier d'une manière objective. L'utilisation du dispositif proposé a pour but de permettre une meilleure définition de la contrainte normale à l'interface et de son évolution au cours du cisaillement.

Après la description de l'appareillage, le principe de l'essai et les premiers résultats obtenus seront présentés. Deux procédures d'essai sont définies : soit des essais à contrainte normale constante avec mesure des variations de volume au cours du cisaillement, soit des essais à volume constant avec mesure de l'évolution de la contrainte normale. Par ailleurs l'arrachement est réalisé soit d'une manière continue, soit par paliers de charge.

Avant de présenter ce travail, certains paramètres susceptibles d'affecter le frottement mobilisé à l'interface seront passés en revue. L'influence de ces paramètres a été déduite soit d'essais réels in situ, soit au laboratoire à l'aide des appareils classiques et sur des modèles réduits. Il est à noter qu'il y a une dépendance entre ces paramètres et que leur importance relative ne fait pas l'unanimité. Malgré cette dépendance les paramètres seront classés comme suit.

\subsection{Paramètres liés à la mise en place de l'inclusion}

Le battage de l'inclusion conduit, soit à une diminution du frottement mobilisé à l'interface surtout dans les sols carbonatés (LE TIRANT et al., 1989), soit à une densification locale qui augmente la valeur de la contrainte normale.
Dans le cas de mise en place par forage et scellement par injection de coulis de ciment, l'adhérence de l'inclusion au sol augmente d'une façon remarquable.

\subsection{Paramètres liés à l'inclusion}

L'état de surface de l'inclusion (lisse ou rugueuse) joue un rôle important sur le frottement mobilisé à l'interface. II est communément admis que les inclusions rugueuses peuvent mobiliser un frottement plus élevé ; en effet pour les inclusions lisses durant le mouvement de la structure au contact du sol, le nombre de grains mis en déplacement est bien plus faible que lorsque la surface est rugueuse ; la contribution de la dilatance et du réarrangement, fonction de ce volume, se trouve sensiblement diminuée. Cependant, les résultats trouvés par différents auteurs concernant ce paramètre ne convergent pas vers une même conclusion ; certains (SCHLOSSER et al., 1979) pensent que le coefficient de frottement (rapport de la contrainte maximale de cisaillement à la contrainte normale appliquée) pour une inclusion lisse peut être pris égal à la moitié de l'angle de frottement interne du sable. YOSHIMI et al. (1981) ont trouvé des coefficients plus faibles en fonction du type de la boîte de cisaillement utilisée.

\subsection{Paramètres liés au sol}

Les remarques essentielles concernant ces paramètres sont récapitulées comme suit:

- le rôle de la densité relative initiale dans le frottement mobilisé à l'interface ne fait pas l'unanimité. Effectivement, DAVIS et PHILIPPONNAT (1978) ont attribué laugmentation du frottement latéral dans le cas des pieux à une compacité initiale plus élevée, la même constatation a été faite par SCHLOSSER et al. (1981), à partir d'essais d'arrachement de bandes métalliques lisses en modèles réduits. Alors que YOSHIMI et al. (1981) considèrent que la densité initiale n'a pas d'effet sur le frottement mobilisé à l'interface et que seules la rugosité et la minéralogie influent sur la résistance au cisaillement à l'interface.

SCHLOSSER et al. (fig. 1) ont constaté que pour les faibles densités, les valeurs maximales du frottement apparent $\mu^{*}$ restent assez voisines du frottement réel $\mu$, alors que pour les fortes densités, les valeurs de $\mu^{*}$ sont nettement supérieures à 1 ;

- en ce qui concerne l'influence de la nature du sol, la forme et les dimensions des grains sont les facteurs prédominants. En effet, une forme plate et anguleuse des grains donne une valeur du frottement latéral maximal plus élevée que celle donnée par des grains fins et arrondis, puisque une forme plate conduit à des surfaces de contact très importantes. SCHLOSSER et al. (1979) ont montré que le coefficient de frottement mobilisé à l'interface sol-inclusion augmente de façon sensible avec l'étalement de la courbe granulométrique. Ce phénomène s'explique par la part élevée de la dilatance que fournit un désenchevêtrement des grains lorsque le frottement maximal est mobilisé. 


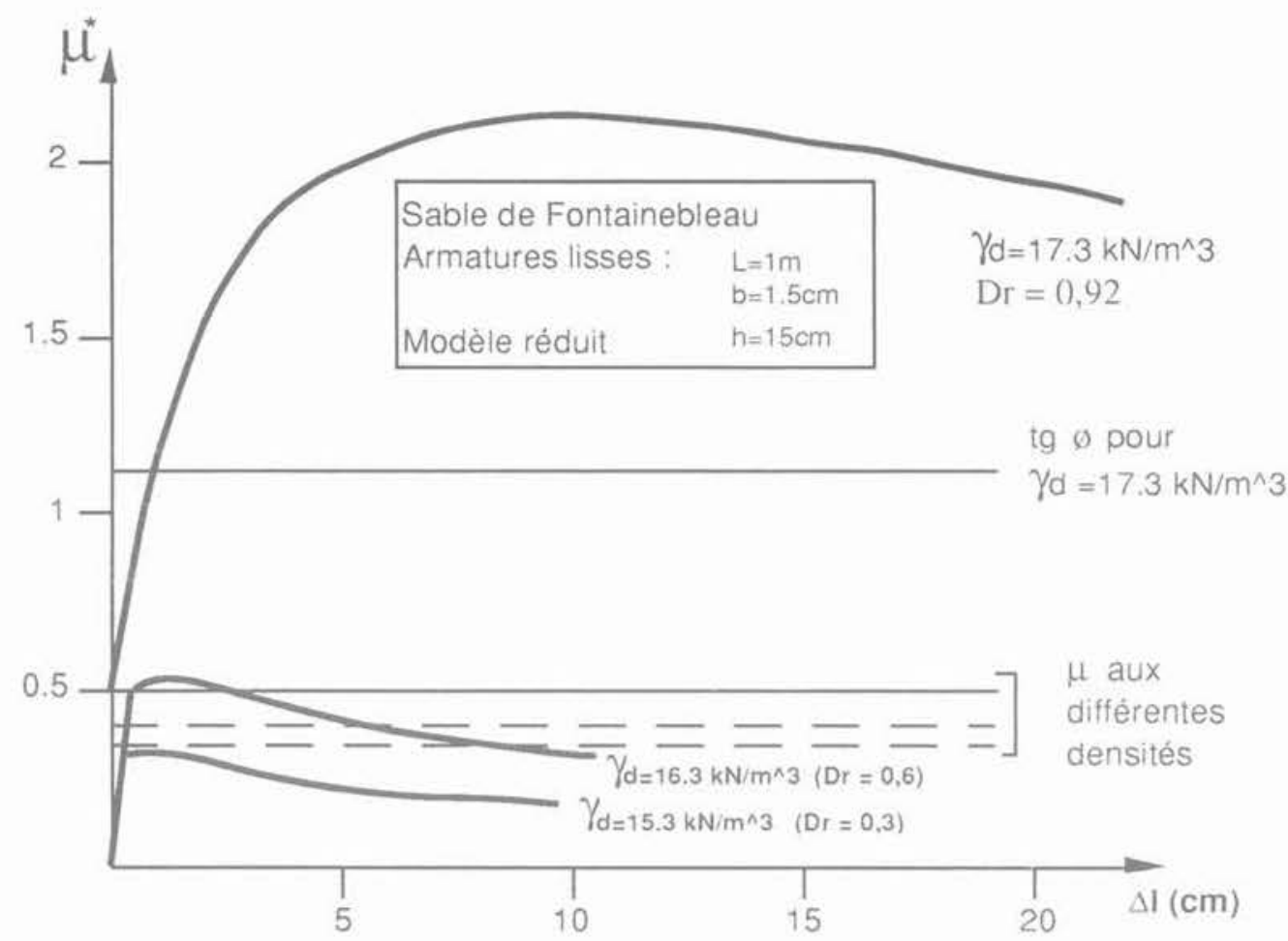

Fig. 1. - Evolution du coefficient de frottement apparent $\mu^{*}$ avec la densité durant un essai d'extraction [SCHLOSSER et al. (1981)], ( $\phi$ étant l'angle de frottement interne du milieu granulaire). Fig. 1. - Evolution of the apparent friction coefficient with

the density during an extraction test [SCHLOSSER and al. (1981)], ( $\phi$ is the internal friction angle of the cohesionless soil).

\section{PROCÉDURES EXPÉRIMENTALES}

Dans ce paragraphe, le dispositif expérimental et le principe de l'essai sont présentés.

\subsection{Dispositif expérimental}

Il est présenté sur la figure 2 et comprend:
- une cuve d'essai constituée d'une buse en béton renforcée par des armatures métalliques extérieures, d'une hauteur de $1 \mathrm{~m}$ et de diamètre intérieur de $0,50 \mathrm{~m}$; cette cuve est remplie de sable sur une hauteur de $0,85 \mathrm{~m}$;

- deux « coussins » gonflants, situés au sommet et à la base de la cuve, permettent l'application d'une contrainte verticale sur le sable;

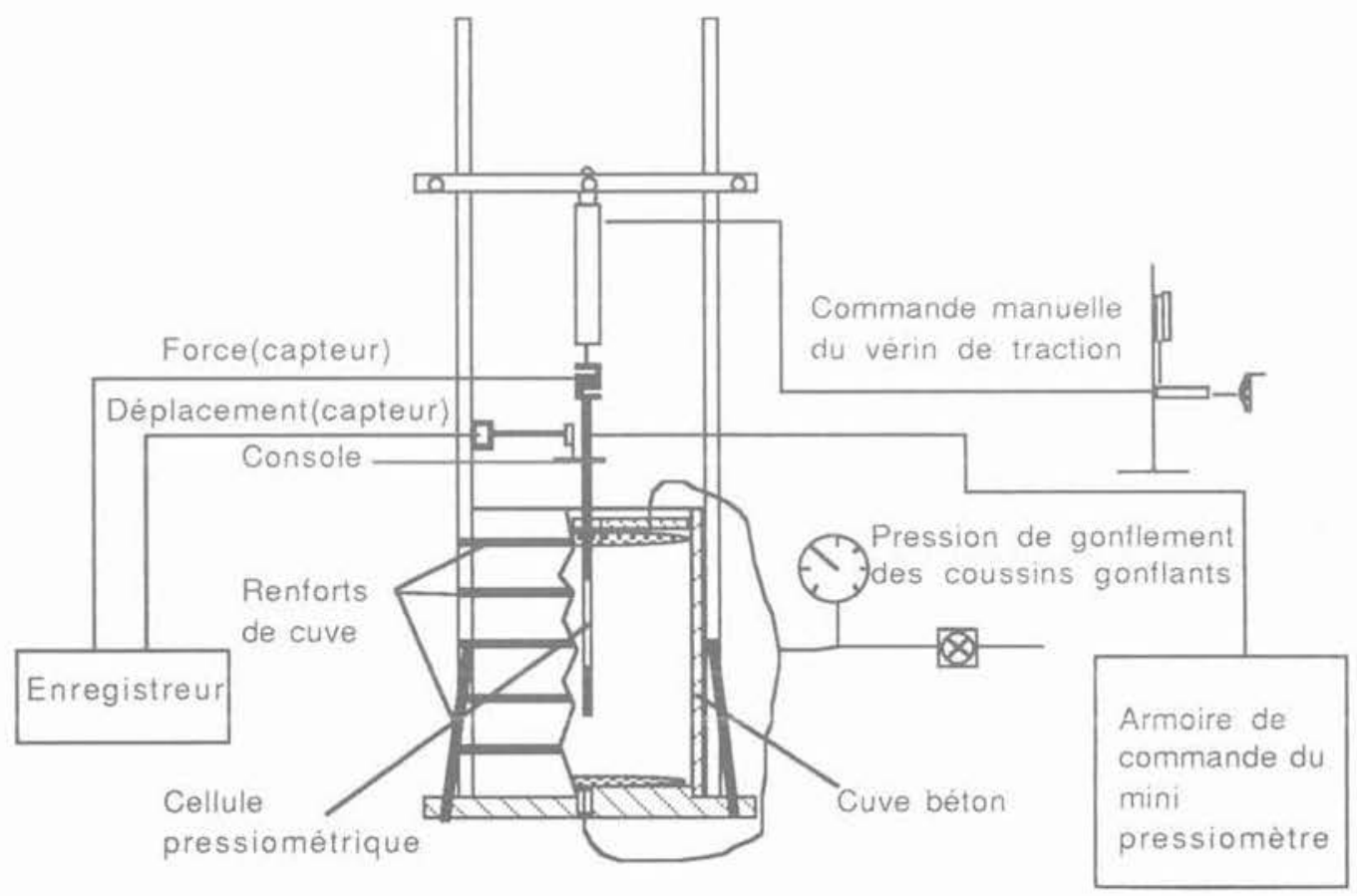

Fig. 2. - Dispositif expérimental des essais d'arrachement progressifs et statiques à l'aide du mini-pressiomètre. Fig. 2. - The apparatus for a progessive and static friction tests. 
- un mini-pressiomètre, type APAGEO, composé d'une pointe à la base, permettant la mise en place d'une sonde lanternée mono-cellulaire, équipée d'un contrôleur pression-volume et de tiges de guidage ; la sonde a un diamètre de $2,5 \mathrm{~cm}$ et une longueur utile de $28,5 \mathrm{~cm}$ (l'état de surface de la sonde peut être lisse ou rugueux)

- un dispositif d'arrachement constitué d'une potence sur laquelle est fixé un vérin permettant l'extraction de la sonde, équipé d'un capteur de force et complété par un dispositif de mesures des déplacements. Les dimensions de la cuve ont été choisies en fonction de celles de la sonde mini-pressiométrique de telle manière que les effets de bord soient évités ou minimisés. Pour cela, on a fait l'inventaire des essais réalisés sur des modèles réduits au laboratoire en vue de déterminer le rapport convenable entre le diamètre de la cuve et celui de la sonde. Les résultats des principales études sont récapitulés dans le tableau 1.

\subsection{Principe de l'essai}

On distingue deux phases durant un essai :

- phase préparatoire;

- essai proprement dit.

La phase préparatoire comprend:

- la mise en place du sable à la densité choisie : les essais préliminaires ont montré la nécessité de reconstituer l'échantillon entre chaque essai pour assurer une bonne reproductibilité comme le montre la figure 3 qui représente les courbes pressiométriques classiques obtenues sur un sable calcaire lors de 5 essais successifs ;

- la mise en place de la sonde soit en moulant le sable autour, soit postérieurement en réalisant un battage ;

- l'application de la contrainte verticale choisie au moyen des coussins dans une gamme de valeurs comprise entre 0 et $100 \mathrm{kPa}$.
Tableau 1. - Dimensions respectives des chambres de calibration et des pressiomètres utilisés lors de deux études.

Table 1. - Relative dimensions of the calibration rooms and of the used pressuremeters.

\begin{tabular}{|c|c|c|}
\hline \multirow{2}{*}{ Auteurs } & \multicolumn{2}{|c|}{ Dimensions (cm) } \\
\cline { 2 - 3 } & $\begin{array}{c}\text { Chambre } \\
\text { triaxiale }\end{array}$ & Pressiomètre \\
\hline \multirow{2}{*}{$\begin{array}{c}\text { Jewell, Fahey } \\
\text { et Wroth (1980) }\end{array}$} & $\begin{array}{c}\mathrm{dc}=100 \\
\text { et hc }=100\end{array}$ & $\begin{array}{c}\mathrm{dp}=8,04 \\
\text { et hp }=50\end{array}$ \\
\cline { 2 - 3 } & \multicolumn{2}{|c|}{$\mathrm{dc} / \mathrm{dp}=12$ et hc/hp $=2$} \\
\hline \multirow{2}{*}{ Fahey (1986) } & $\begin{array}{c}\mathrm{dc}=40 \\
\text { et hc }=20\end{array}$ & dp $=4$ \\
\cline { 2 - 3 } & \multicolumn{2}{|c|}{$\mathrm{dc} / \mathrm{dp}=10$ et hc/hp $=1$} \\
\hline
\end{tabular}

Avec : - dc : diamètre de la chambre de calibration : - hc: hauteur de la chambre de calibration;

- $\mathrm{dp}$ : diamètre du pressiomètre :

- hp : hauteur du pressiomètre.

Pour l'essai proprement dit, on commence par l'application de la pression interne dans le mini-pressiomètre et on attend la stabilisation des déformations; les essais d'étalonnage de la sonde sont réalisés avant et après chaque mesure. La contrainte normale à l'interface est calculée en fonction de la pression interne appliquée et de la raideur propre de la sonde.

L'arrachement par traction est réalisé suivant deux procédures distinctes :

- arrachement progressif à vitesse constante (vitesse de déplacement de $0,65 \mathrm{~mm} / \mathrm{s}$ ) avec enregistrement de la courbe effort-déplacement. Nous rappelons que PHILIPPONNAT (1986) durant les essais au phicomètre a réalisé lui aussi des arrachements progressifs à l'aide d'une sonde monocellulaire pour déterminer la résistance au cisaillement des sols en place;

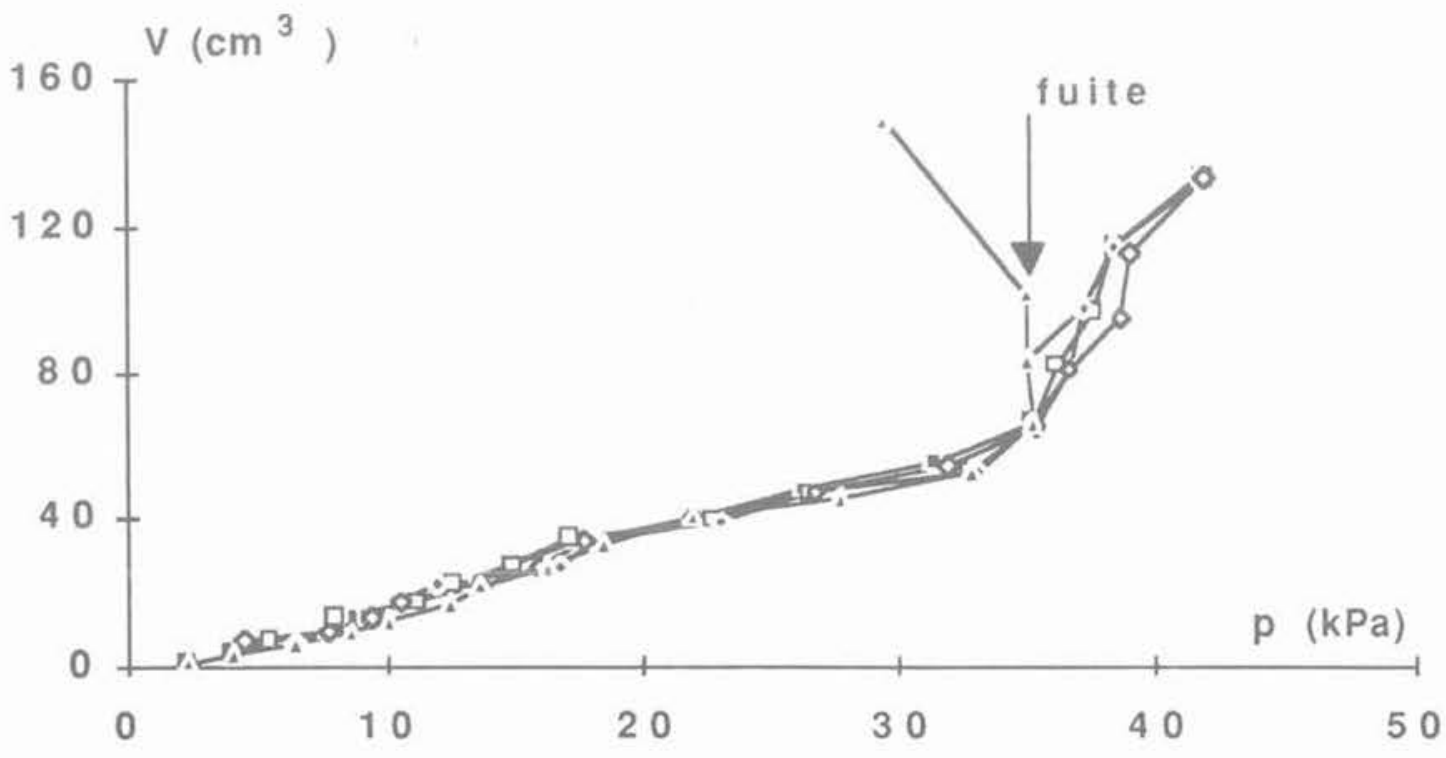

Fig. 3. - Courbes pressiométriques classiques sur le sable calcaire à l'état lâche. (Essais de reproductibilité). Fig. 3. - Classical pressuremeter's curves (foose calcareous sand). (Reproductible tests). 
- arrachement par paliers de chargement croissant avec mesure des déformations en fonction du temps pour chaque palier. Pour ces essais, on a suivi le projet du mode opératoire du LCPC (1970) et le Projet National CLOUTERRE (1986-1990).

Enfin et seulement dans le cas de l'arrachement progressif, nous avons utilisé les deux procédures décrites plus haut ;

- cisaillement à contrainte normale constante et mesure des variations de volume:

- cisaillement à volume constant et mesure des variations de la contrainte normale.

Il faut noter que lors de la mise en pression de la sonde, celle-ci ne conserve pas une forme exactement cylindrique mais devient légèrement ovoïde, cependant le rapport entre la longueur utile de la sonde et son diamètre est très élevé et par conséquent ce défaut reste a priori peu important. De plus, lors de la majeure partie des essais (comme cela sera indique plus loin) l'effort maximum d'arrachement est obtenu pour un déplacement très faible (en général inférieur à $2 \mathrm{~mm}$ ) et reste constant pour un déplacement atteignant $10 \mathrm{~mm}$. C'est pourquoi on peut penser que l'existence d'une butée et son effet sur la mesure ont un poids faible.

\section{DESCRIPTION DES SOLS UTILISÉS}

Nous avons utilisé deux types de sable, un sable siliceux et un sable calcaire. Le sable siliceux provient des alluvions de la Moselle et le sable calcaire correspond à une formation de pente du type grouine* . Dans les deux cas, nous avons réalisé un tamisage préalable de manière à obtenir des compositions granulométriques constantes et reproductibles.

Le tableau 2 résume les caractéristiques granulométriques des deux sables utilisés et donne les valeurs des densités limites obtenues dans chaque cas. Il s'agit de deux sables propres mal gradués.

\section{EFFET DU CHEMIN DE CONTRAINTES}

Une étude de linterface a été préalablement conduite en utilisant les appareils classiques du laboratoire (boîte de cisaillement direct et appareil de cisaillement annulaire par torsion) (ABDERRAHIM, 1991). Durant cette étude, on a réalisé des essais à contrainte normale constante et des essais à volume constant. De la même manière, le nouveau dispositif a permis la réalisation des essais selon les mêmes chemins de contraintes. Les essais à contrainte normale constante donnent la résistance au cisaillement à linterface et les essais à volume constant permettent d'étudier le comportement à l'interface sol-inclusion et tiennent compte de ce qui se passe réellement in situ.

Les deux types de sables décrits ci-dessus ont été utilisés ainsi que différentes conditions expérimentales relatives à la rugosité de l'inclusion et son mode de mise en place ainsi qu'à la densité relative. La rugosité des feuilles d'acier qui enveloppent la sonde a été prise de la façon suivante : tout d'abord elles ont été prises parfaitement lisses, ensuite, on y a collé le même sable que celui utilisé pendant l'essai.

Dans ce paragraphe, des résultats correspondants aux deux procédures définies, utilisées lors de l'arrachement progressif́ sont présentés.

\subsection{Arrachement à contrainte normale constante}

Durant la première série d'essais, la même contrainte normale initiale à l'interface a été imposée pour différentes contrainte verticales appliquées. Les courbes correspondantes à ces essais sont représentées dans la figure 4.

Pour les trois essais réalisés dans un échantillon lâche, des contraintes normales initiales très voisines $(83,36$; 84,$4 ; 84,47 \mathrm{kPa}$ ) ont été imposées. Les trois courbes en pointillé correspondantes à ces contraintes montrent que le frottement ultime mobilisé est le même. On constate par contre que la variation de volume diminue lorsque la contrainte verticale appliquée augmente. Bien que la pression interne appliquée reste constante, la variation du volume de la sonde, durant le cisaillement, entraîne du fait de la raideur propre de la sonde une légère variation de la contrainte normale réelle ( 3 à $5 \mathrm{kPa}$ ), mais ce phénomène est impossible à maîtriser. Dans la même figure est portée la courbe d'un essai réalisé dans un échantillon à l'état dense; on remarque que le sable se contracte très peu durant l'arrachement et par conséquent la contrainte normale effectivement appliquée reste alors pratiquement constante.

\footnotetext{
- Grouine (appelation locale) : formation en éboulis au pied d'une falaise calcaire, accumulée dans des conditions périglaciaires. Les débris de roches sont généralement anguleux, disposés en lits obliques alternant avec des passées de matériel fin plus argileux.
}

Tableau 2. - Caractéristiques physiques de nos deux sables ( $D$ en $\mathrm{mm}$ ).

Table 2. - Physical characteristics of our two sands.

\begin{tabular}{|c|c|c|c|c|c|c|c|c|}
\hline Sable & Composition & \multicolumn{9}{|c|}{ Caractéristiques } \\
\hline & minéralogique & D10 & D30 & D60 & Cu & Cc & $\gamma$ min $\left(\mathrm{kN} / \mathrm{m}^{3}\right)$ & $\gamma \mathrm{max}\left(\mathrm{kN} / \mathrm{m}^{3}\right)$ \\
\hline Toul & $96(\%) \mathrm{CaCO}_{3}$ & 0,4 & 0,8 & 1,36 & 3,4 & 1,17 & 12,0 & 14,1 \\
\hline Moselle & Siliceux & 0,35 & 0,5 & 0,8 & 2,29 & 0,89 & 14,5 & 17,2 \\
\hline
\end{tabular}




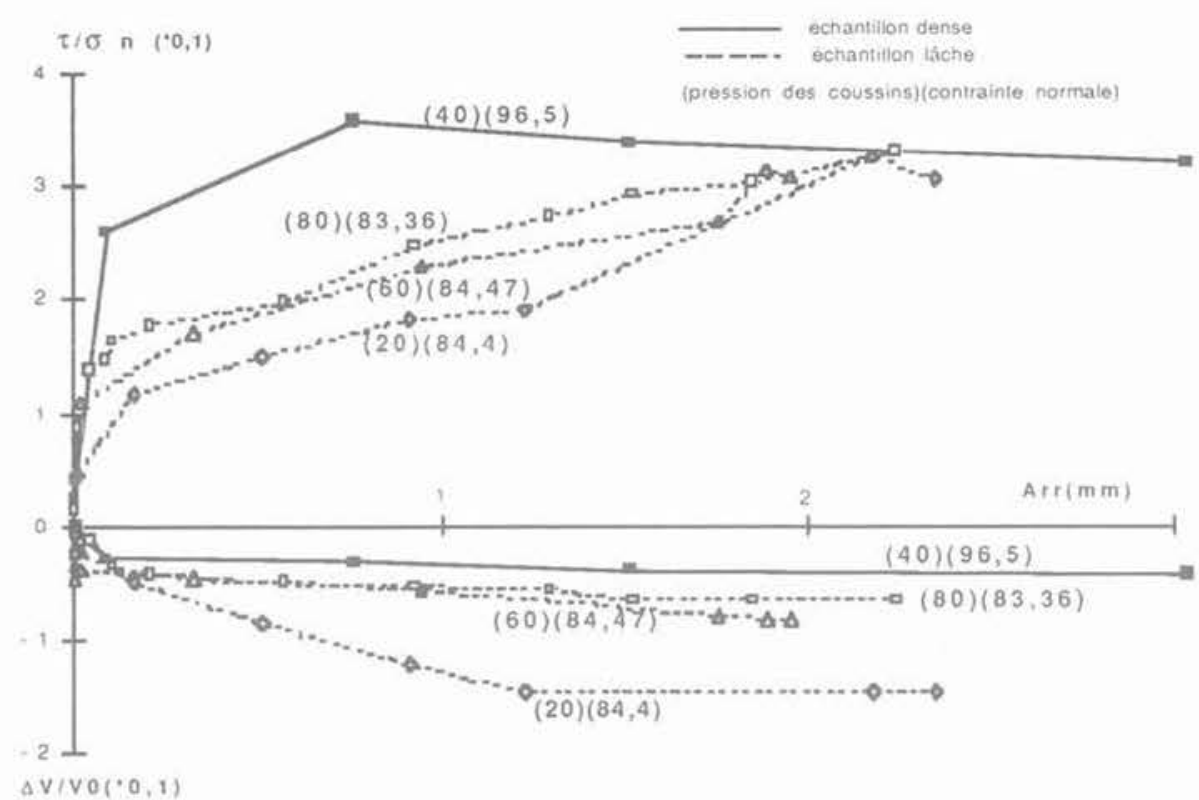

Fig. 4. - Evolution de $\pi / \sigma_{n}$ à l'interface sable siliceux-sonde lisse et variation du volume de la sonde avec l'arrachement; (trois essais à l'état lâche et un essai à l'état dense.

On a indiqué sur chaque courbe la valeur de la contrainte verticale appliquée et la valeur de la contrainte normale en $k$ Pa).

Fig. 4, - Evolution of $\pi / \sigma_{n}$ at the interface siliceous sand-smooth probe and volumic variation during shearing.

Durant la deuxième série d'essais, des arrachements progressifs à différentes contraintes normales dans un sable lâche ont été réalisés. La sonde lisse a été mise en place soit par moulage, soit par battage. D'après les courbes des figures $5 a$ et $5 b$, on constate que la courbe de l'évolution du coefficient de frottement (rapport de la contrainte tangentielle sur la contrainte normale: $\tau\left(\sigma_{n}\right)$ en fonction du déplacement est assez raide ; le maximum est obtenu pour un déplacement de $2 \mathrm{~mm}$ environ et au-delà le rapport $\tau / \sigma_{\mathrm{n}}$ présente un palier jusqu'à des valeurs de déplacement supérieures à $8 \mathrm{~mm}$.
On constate que le mode de mise en place n'a pas d'influence sur les résultats obtenus.

BOULON et al. (1986), durant leurs essais à l'aide de l'appareil de cisaillement direct à rigidité imposée, ont trouvé des courbes de même allure.

\subsection{Arrachement à volume constant}

Lors d'un arrachement à volume constant, la contrainte normale appliquée chute d'une manière très importante et pour un déplacement faible (de l'ordre de $0,5 \mathrm{~mm}$ comme le montre la figure 6) atteint un

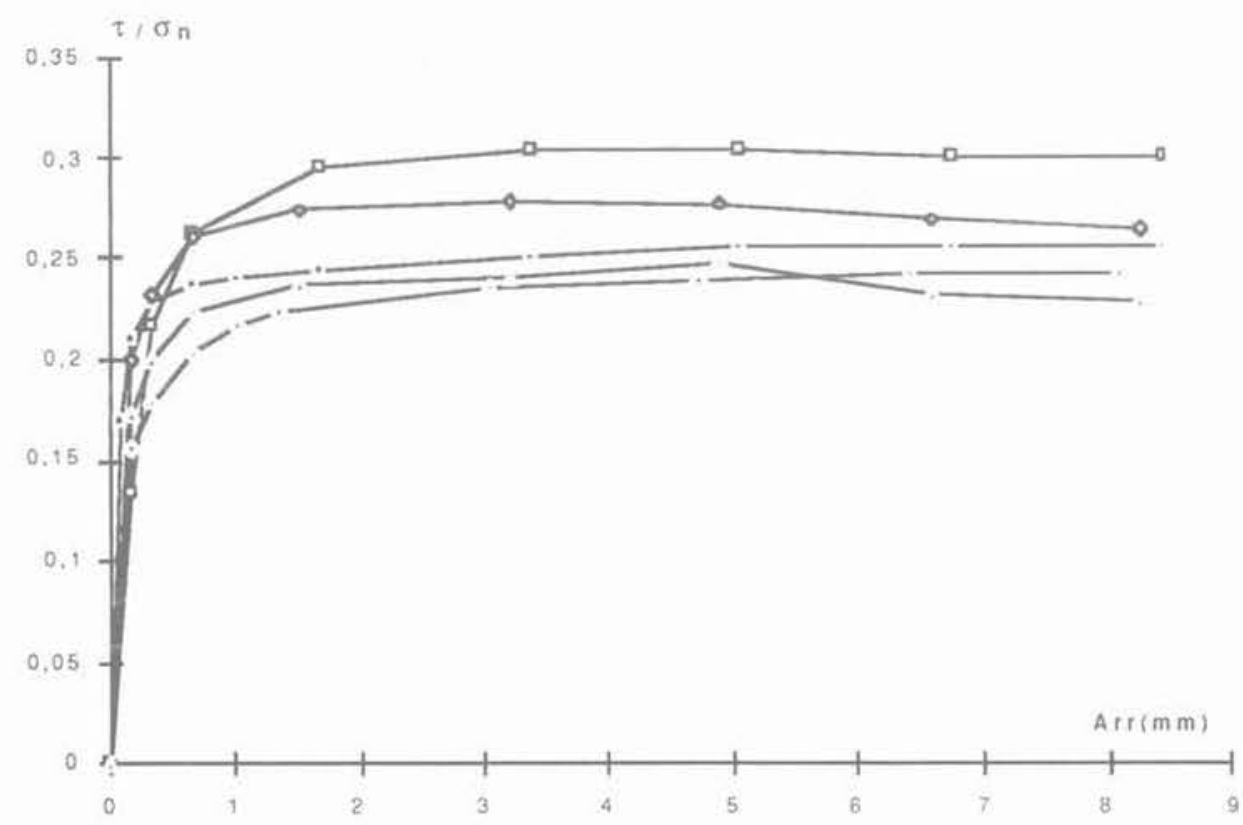

Fig. 5a, - Courbes d'arrachement de la sonde mini-pressiométrique (lisse) introduite par moulage dans le sable siliceux à l'état lâche.

Fig. 5a. - Friction curves of the smooth pressuremetric probe introduced by moulding in the loose siliceous sand. 


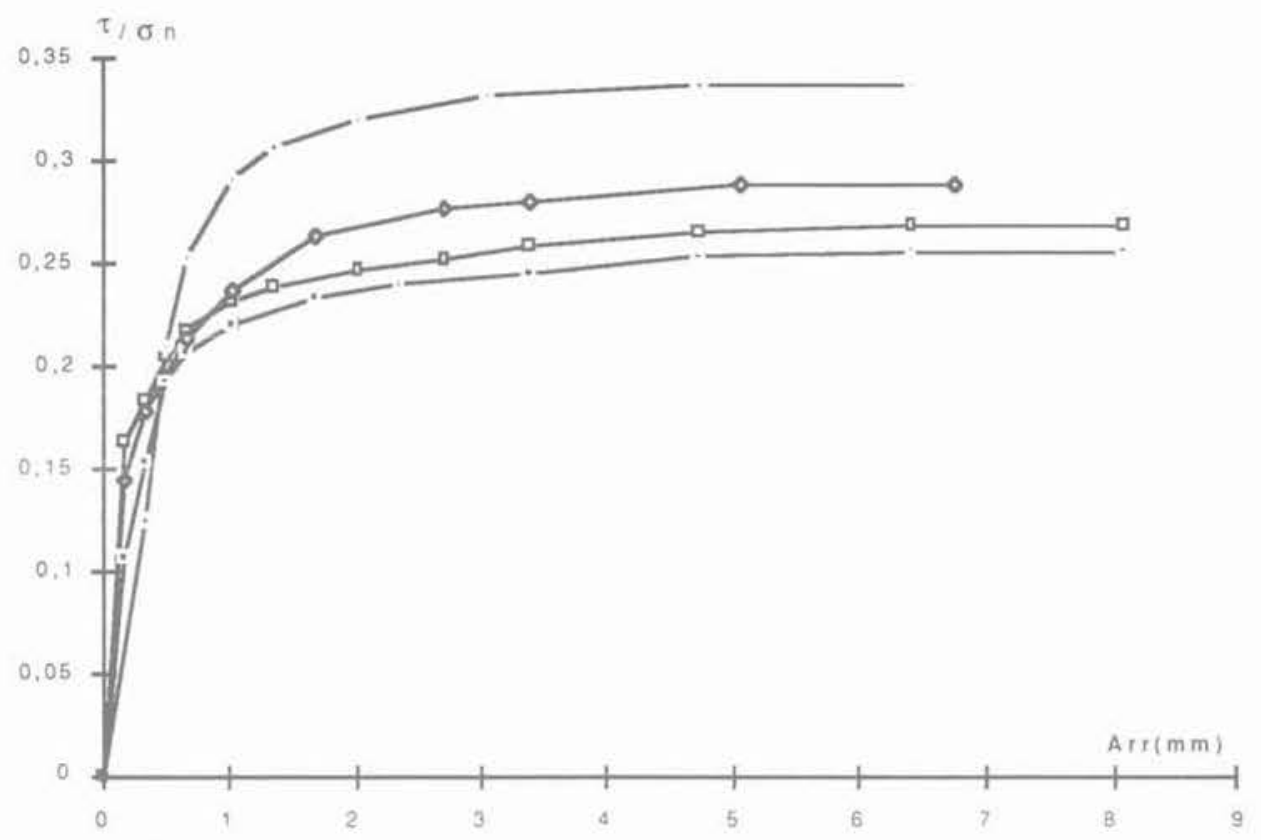

Fig. 5b. - Courbes d'arrachement de la sonde mini-pressiométrique (lisse) introduite par battage dans le sable siliceux à l'état lấche.

Fig. 5b. - Friction curves of the smooth pressuremetric probe introduced by driving in the loose siliceous sand.

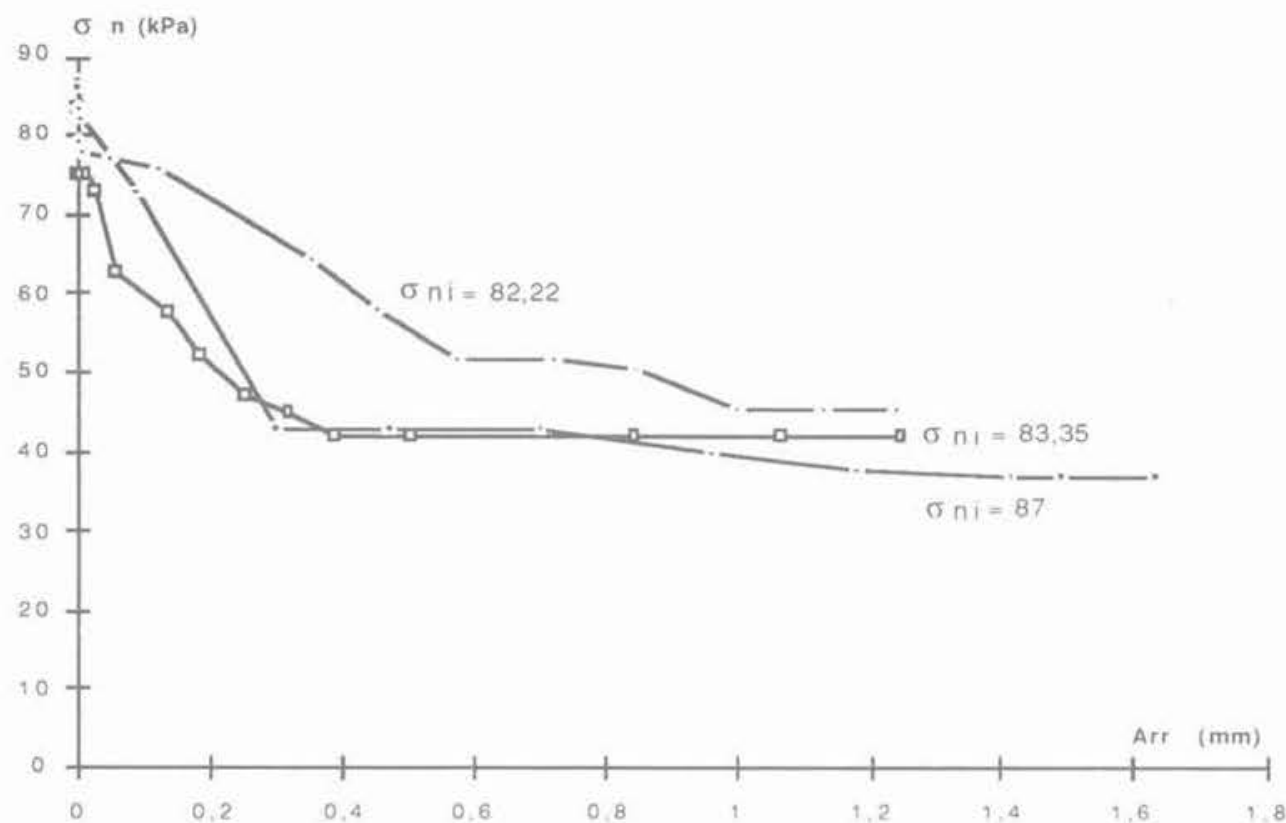

Fig. 6. - Variation de la contrainte normale (en kPa) avec l'arrachement d'une sonde lisse introduite par moulage dans un sable siliceux à l'état lâche (essais à volume constant).

Fig. 6. - A normal stress variation during the friction of a smooth probe introduced by moulding in a loose siliceous sand (constant volume tests)

palier dont la valeur est de l'ordre de la moitié de la contrainte initiale appliquée, pour une sonde lisse et lorsque le sol est dans un état lâche. Par contre SCHLOSSER et GUILLOUX (1981) lors d'arrachement d'une inclusion lisse ont obtenu des résultats différents indiquant au contraire une augmentation de la contrainte normale.
La diminution de la contrainte normale durant le cisaillement à volume constant apparaît sur les chemins de contraintes représentés sur la figure 7 .

Le coefficient de frottement à l'interface à volume constant atteint sa valeur la plus élevée pour un déplacement de l'ordre de $1 \mathrm{~mm}$ (fig. 8). Ce dépla- 


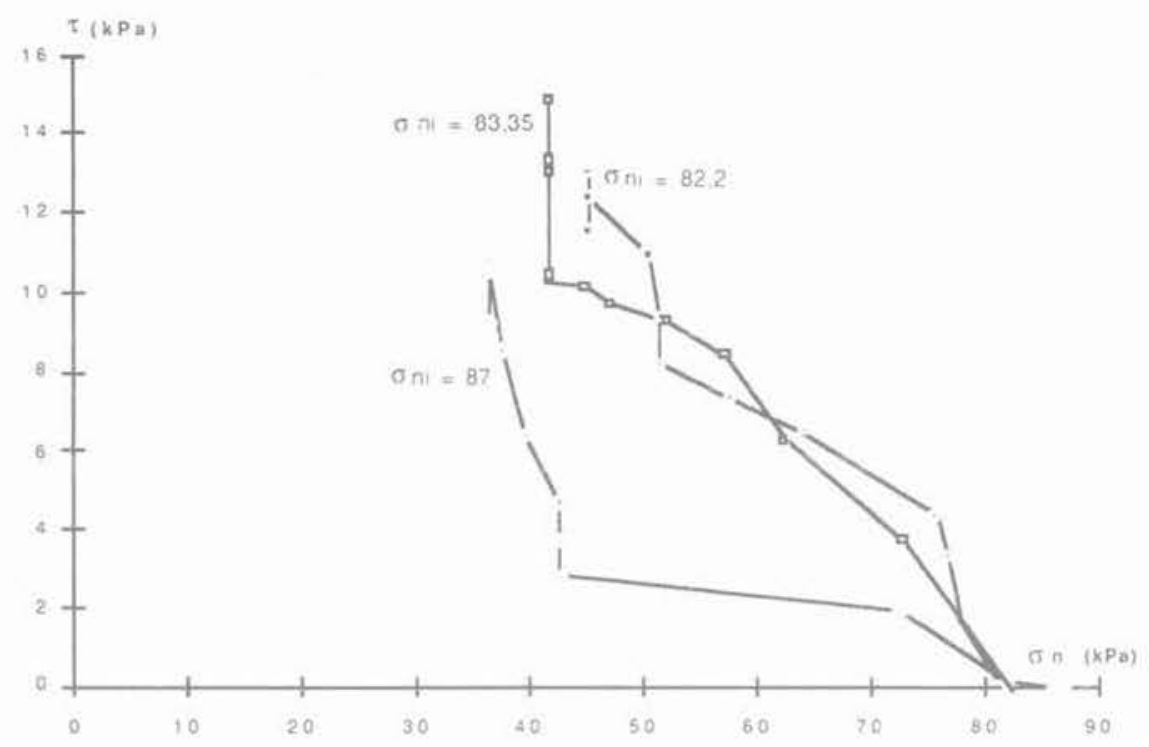

Fig. 7. - Chemins de contrainte durant un essai à volume constant.

Fig. 7. - Stress paths during a constant volume test.

cement est légèrement plus faible que celui qui correspond à la mobilisation de la valeur stabilisée lors des essais à contrainte normale constante (fig. $5 a$ et $5 b$ ).

$\mathrm{Si}$ on compare maintenant (fig. 9) les valeurs du coefficient de frottement réel mobilisé à l'interface lors des deux types d'essais, on constate que pour des conditions identiques de mise en place, de rugosité de la sonde et d'état du sol, les résultats obtenus sont proches.

Généralement pour la terre armée ou les clous, les concepteurs utilisent un coefficient de frottement apparent noté $\mu^{*}$ qui est égal à $\tau / \sigma_{\mathrm{n}}$ avec :

- linclusion $\sigma_{n}$ : contrainte horizontale entourant lnclusion dont la valeur exacte est ignorée et prise traditionnellement égale à $k_{0} \sigma_{v}\left(k_{0}\right.$ : coefficient de pression au repos des terres, $\sigma_{v}$ : contrainte verticale) ;

- $\tau$ : contrainte de cisaillement correspondante.

Si l'on admet que la contrainte horizontale chute, comme nous l'avons noté pendant nos essais, cela signifie que le coefficient de frottement réel est environ deux fois plus élevé que le coefficient de frottement apparent. L'intérêt de la mesure proposée dans ce cas serait de bien appréhender la contrainte horizontale réelle; ce qui permettrait alors, en admettant que le coefficient de frottement réel soit déterminé exactement, d'apporter une amélioration au dimensionnement.

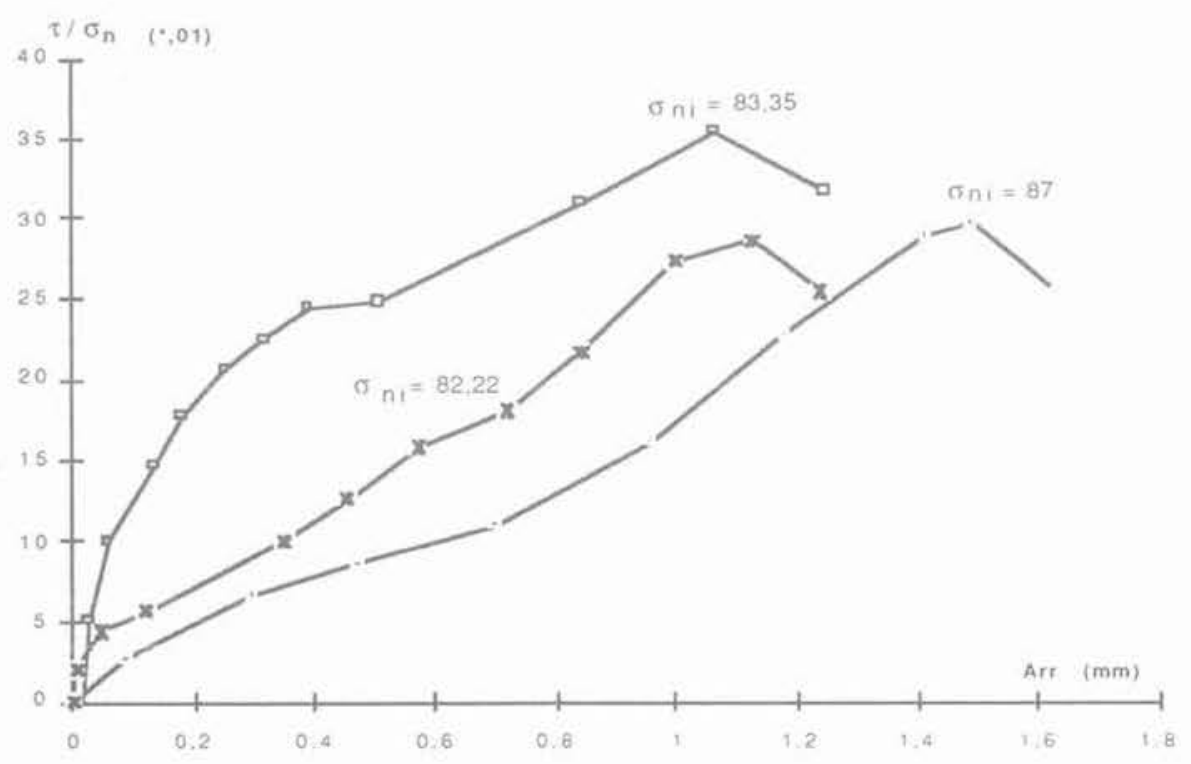

Fig. 8. - Evolution du rapport r/on avec l'arrachement d'une sonde lisse introduite par moulage dans un sable siliceux à l'état lâche à volume constant (contrainte en $\mathrm{KPa}$ ).

Fig. 8. - Evolution of t/on during shearing at the interface loose siliceous sand-smooth probe during a constant volume test (stress in $\mathrm{kPa}$ ). 


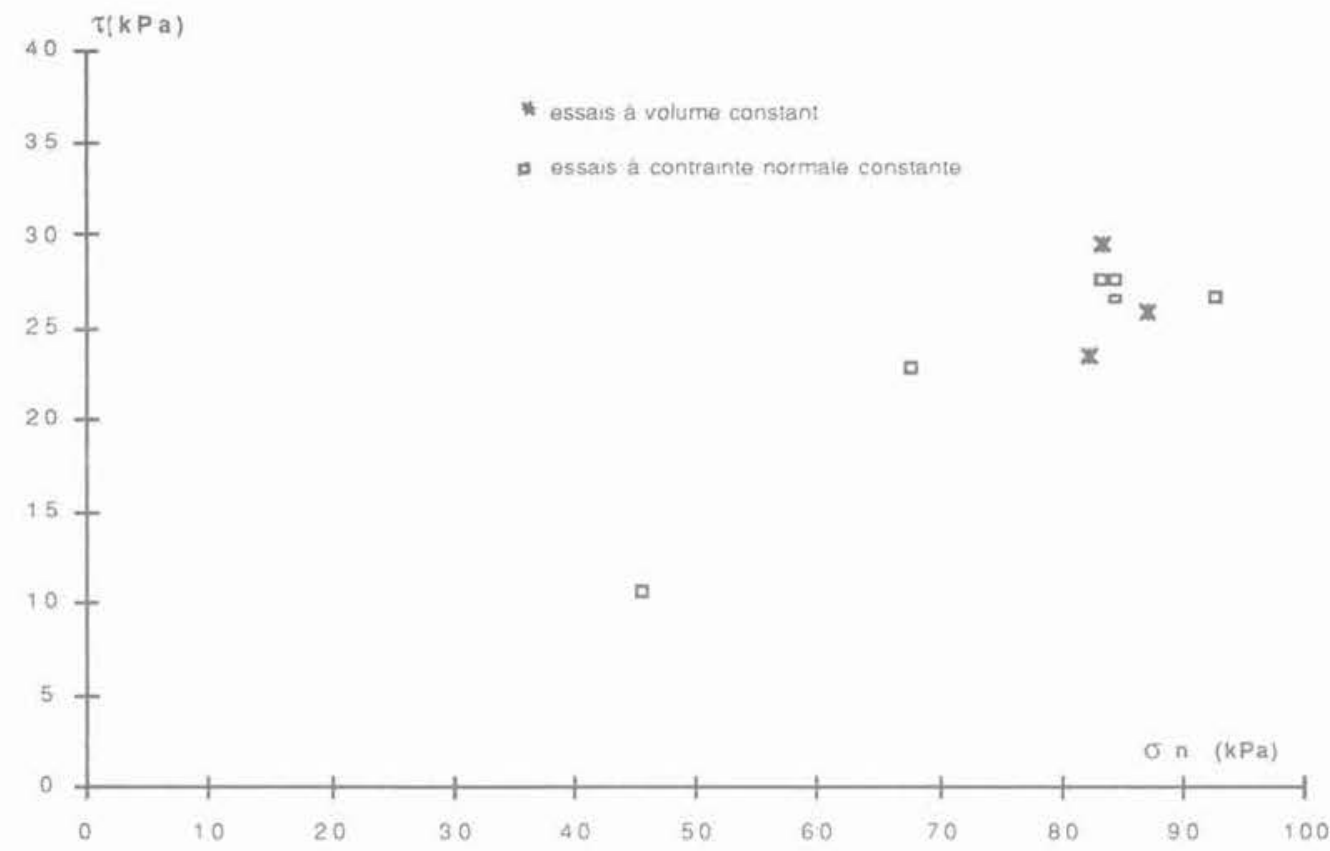

Fig. 9. - Courbe du frottement à l'interface sable siliceux à l'état lâche-sonde lisse introduite par moulage pour des essais à on constante et des essais à volume constant.

Fig. 9. - Friction curve at the interface loose siliceous sand-smooth probe introduced by moulding from constant stress tests and constant volume tests.

\section{ESSAIS D'ARRACHEMENT PAR PALIERS}

il s'agit d'arrachement à contrainte normale constante. $\mathrm{Si}$ les deux types d'essais d'arrachement (progressif ou par paliers) permettent de déterminer l'effort d'arrachement maximal que peut supporter une inclusion, l'arrachement par paliers permet de déterminer aussi un effort de fluage correspondant à une augmentation importante des déformations différées.

La description de l'essai d'arrachement par paliers est faite au moyen de trois types de graphiques.

Le premier (fig. 10) donne l'évolution de l'effort d'arrachement en fonction des déplacements cumulés.

Le second (fig. 11) représente pour chacun des paliers de chargements, l'évolution de la déformation en fonction du logarithme décimal du temps : les courbes obtenues sont des droites dites droites de stabilisation caractérisées par leurs pentes. On a indiqué pour chaque palier le rapport de l'effort d'arrachement (au palier) sur l'effort maximum.

Le troisième (fig. 12) montre les valeurs des pentes des droites de stabilisation en fonction du pourcentage de l'effort d'arrachement maximum, il permet la détermination d'une charge de fluage qui correspond à une augmentation importante des pentes de ces droites.

Enfin on a représenté (fig. 13) les valeurs du rapport charge de fluage (Qf) sur charge limite $\left(\mathrm{Q}^{\text {st }}\right.$ max) pour l'ensemble des essais d'arrachement par paliers qui ont été réalisés (soit sur le sable calcaire, soit sur le sable siliceux) en utilisant les deux types de son- des et différentes contraintes normales à l'interface. La valeur moyenne obtenue est de $60 \%$, mais il existe une dispersion importante; cette dispersion n'étant pas liée apparemment aux conditions d'essais. Il est vraisemblable qu'une augmentation du nombre de paliers permettrait d'affiner la détermination de la charge de fluage.

\section{COMPARAISON DES DEUX MODES D'ARRACHEMENT}

En ce qui concerne le déplacement nécessaire pour obtenir le maximum de l'effort d'arrachement, on constate que l'arrachement par paliers conduit à une valeur plus élevée que l'arrachement progressif $\left(Q^{\text {prog }} \max \right)$; ce qui est logique puisque dans le premier cas on permet le développement des déformations différées. Par contre (fig. 14) les valeurs d'effort limite mesurées par les deux méthodes conduisent, toutes choses égales par ailleurs, à des résultats quasiment identiques. L'avantage de la méthode par paliers réside dans la mise en évidence de la charge de fluage.

\section{CONCLUSION}

Les premiers résultats obtenus ici montrent la fiabilité de l'expérimentation et répondent d'une façon satisfaisante à nos objectifs initiaux à savoir une meilleure connaissance des phénomènes à l'interface, et une mesure directe du coefficient de frottement. 


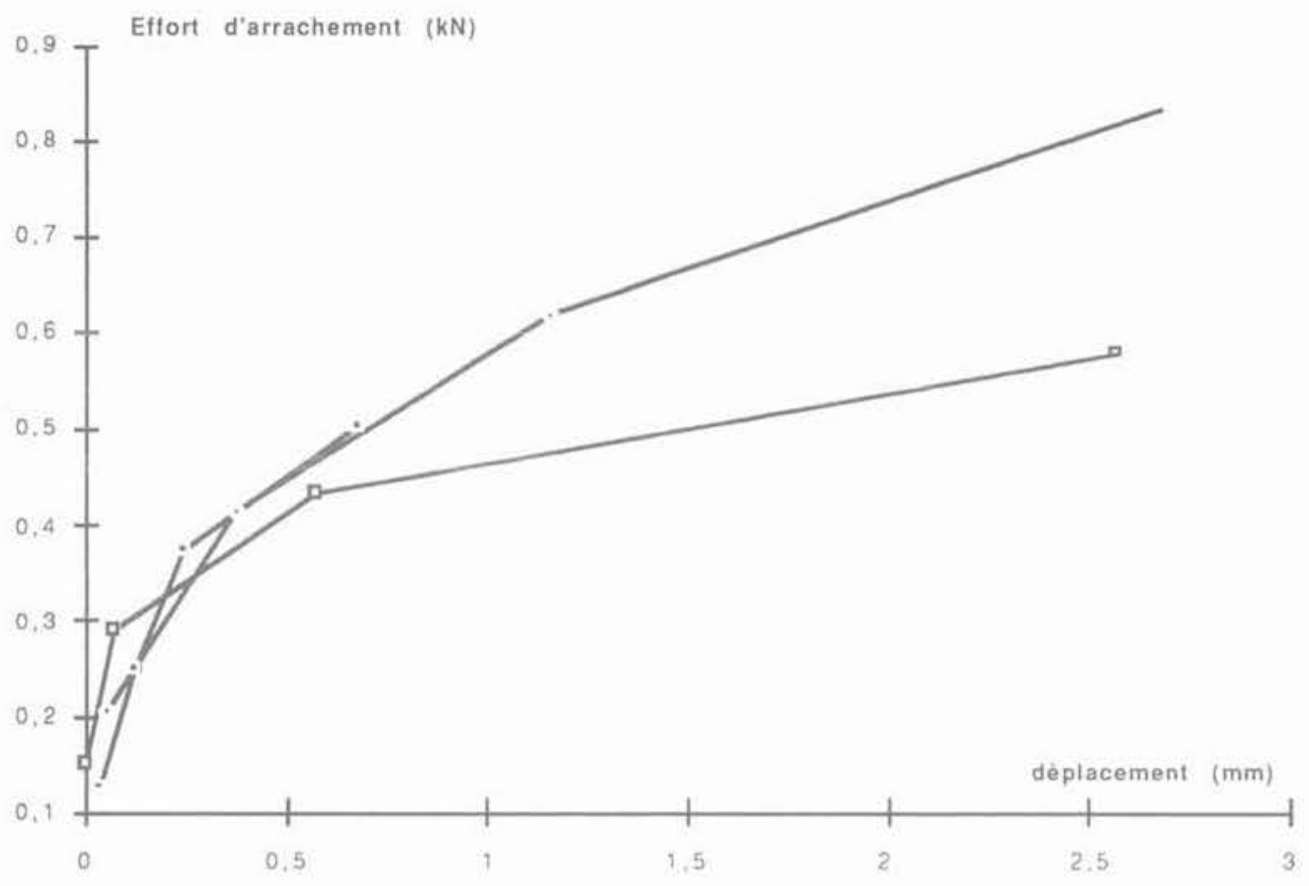

Fig. 10. - Courbes effort de traction-arrachement pour différentes contraintes normales. La sonde rugueuse est introduite dans un sable siliceux à l'état lâche par moulage, (la pression appliquée à l'aide des coussins est de $50 \mathrm{kPa}$ ).

Fig. 10. - Traction effort as a fonction of friction for different pressure into the probe lloose siliceous sand-rough probe, vertical stress is equal to $50 \mathrm{kPa}$ ).

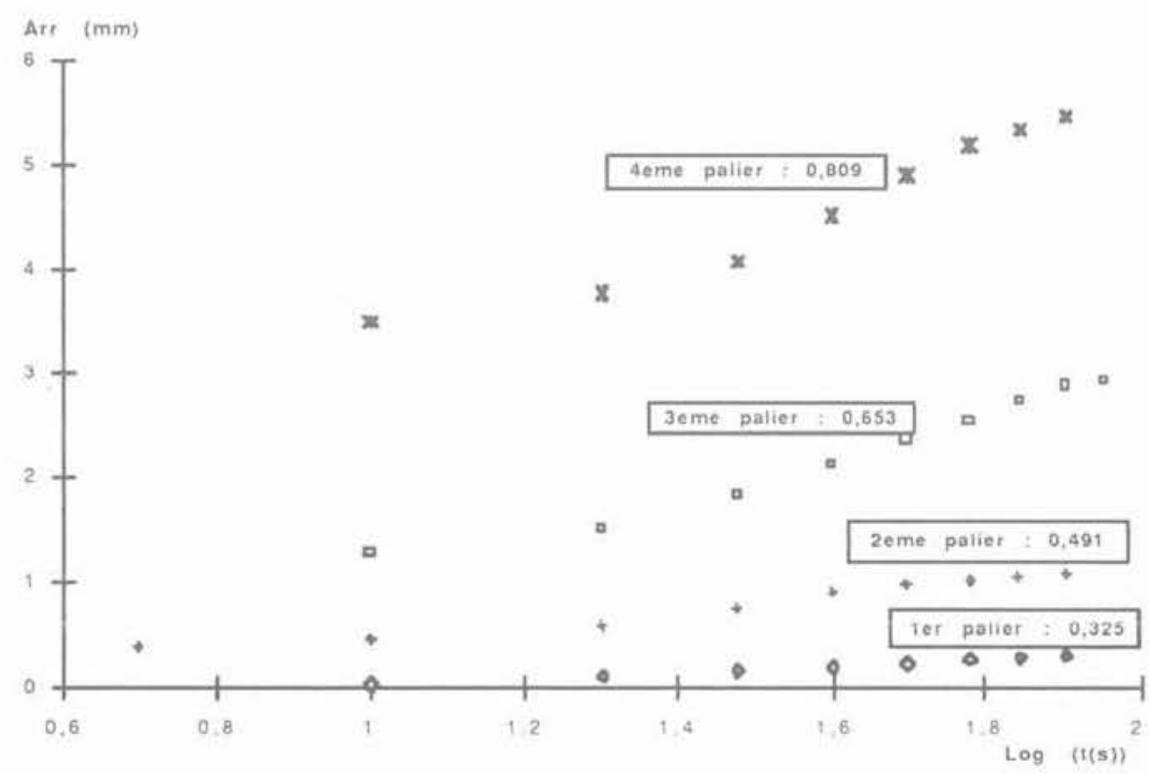

Fig. 11. - Courbes de stabilisation à l'interface sable siliceux à l'état lâche-sonde mini-pressiométrique rugueuse.

Fig. 11. - Stabilization curves at the interface loose siliceous sand-rough probe. 


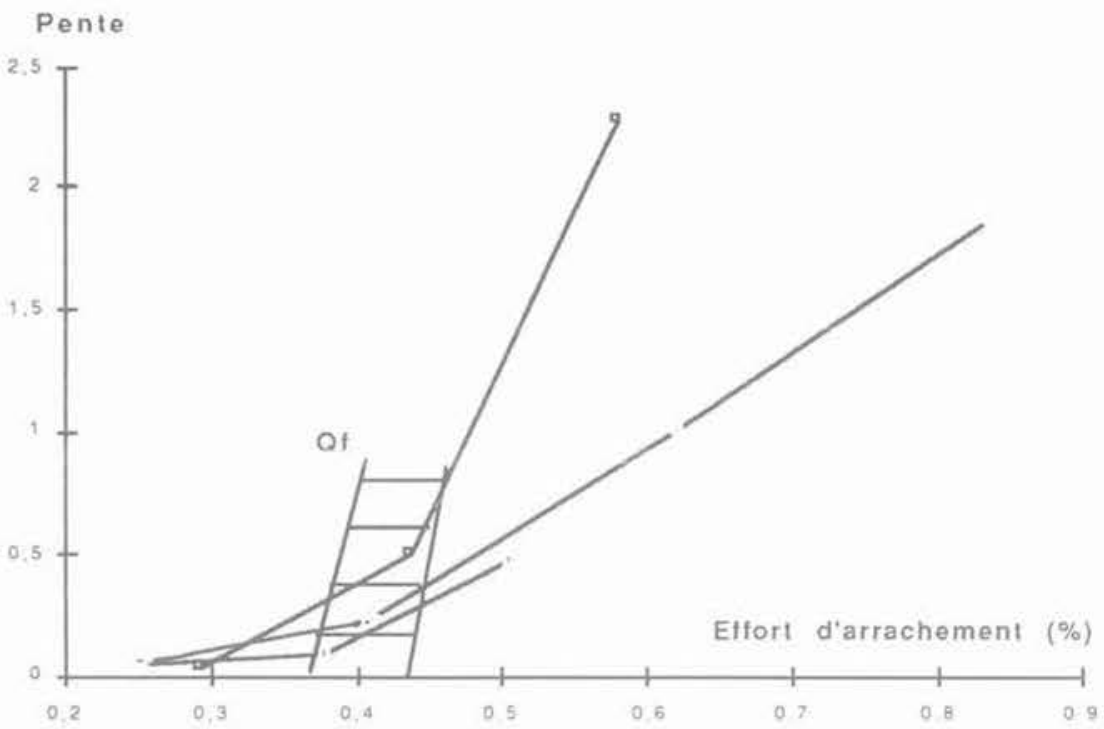

Fig. 12. - Courbes effort de traction-pentes des droites de stabilisation pour différentes contraintes normales ; la sonde rugueuse est introduite dans un sable siliceux à l'état lâche par moulage, (la pression appliquée à l'aide des coussins est de $50 \mathrm{kPa}$ ).

Fig. 12. - Curves effort of traction-slope of the straights of stabilization for different pressures into the probe lloose siliceous sand-rough probe, vertical stress is equal to $50 \mathrm{kPa}$.

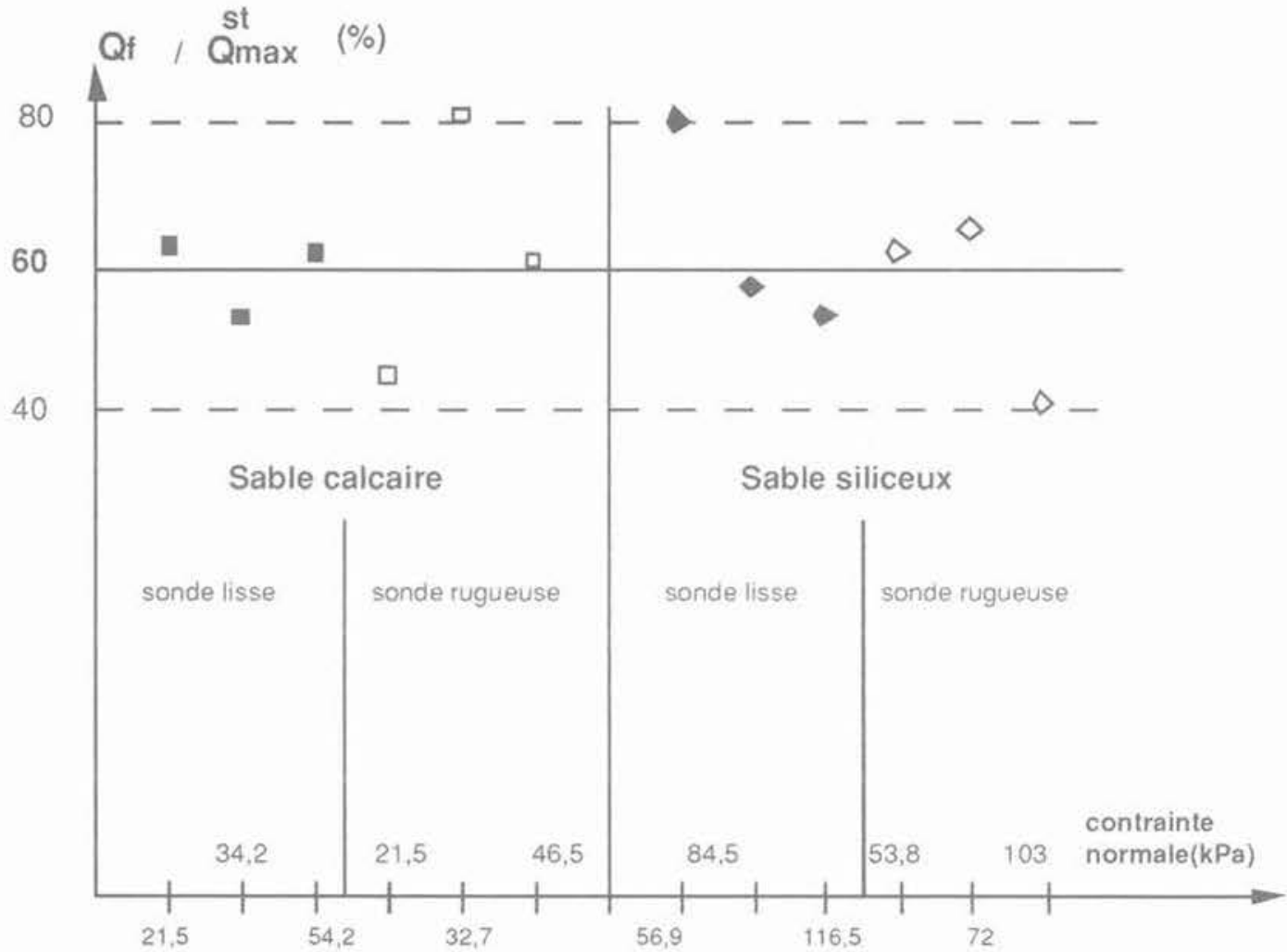

Fig. 13. - Rapport de l'effort de fluage sur l'effort limite durant un essai d'arrachement par paliers selon le type de sable et la rugosité de la sonde mini-pressiométrique $\left(\sigma_{v}=50 \mathrm{kPa}\right)$.

Fig: 13. - Ratio of the kreep effort on the limit effort during a static friction test

for different roughness of the probe and different kind of sands $\left(\sigma_{v}=50 \mathrm{kPa}\right)$.

Les valeurs limites des efforts d'arrachement, à contrainte normale constante, sont indépendantes du mode d'arrachement (progressif ou par paliers).
Les essais à volume constant ont permis de mettre en évidence la chute importante de la contrainte normale à l'interface durant le cisaillement. 


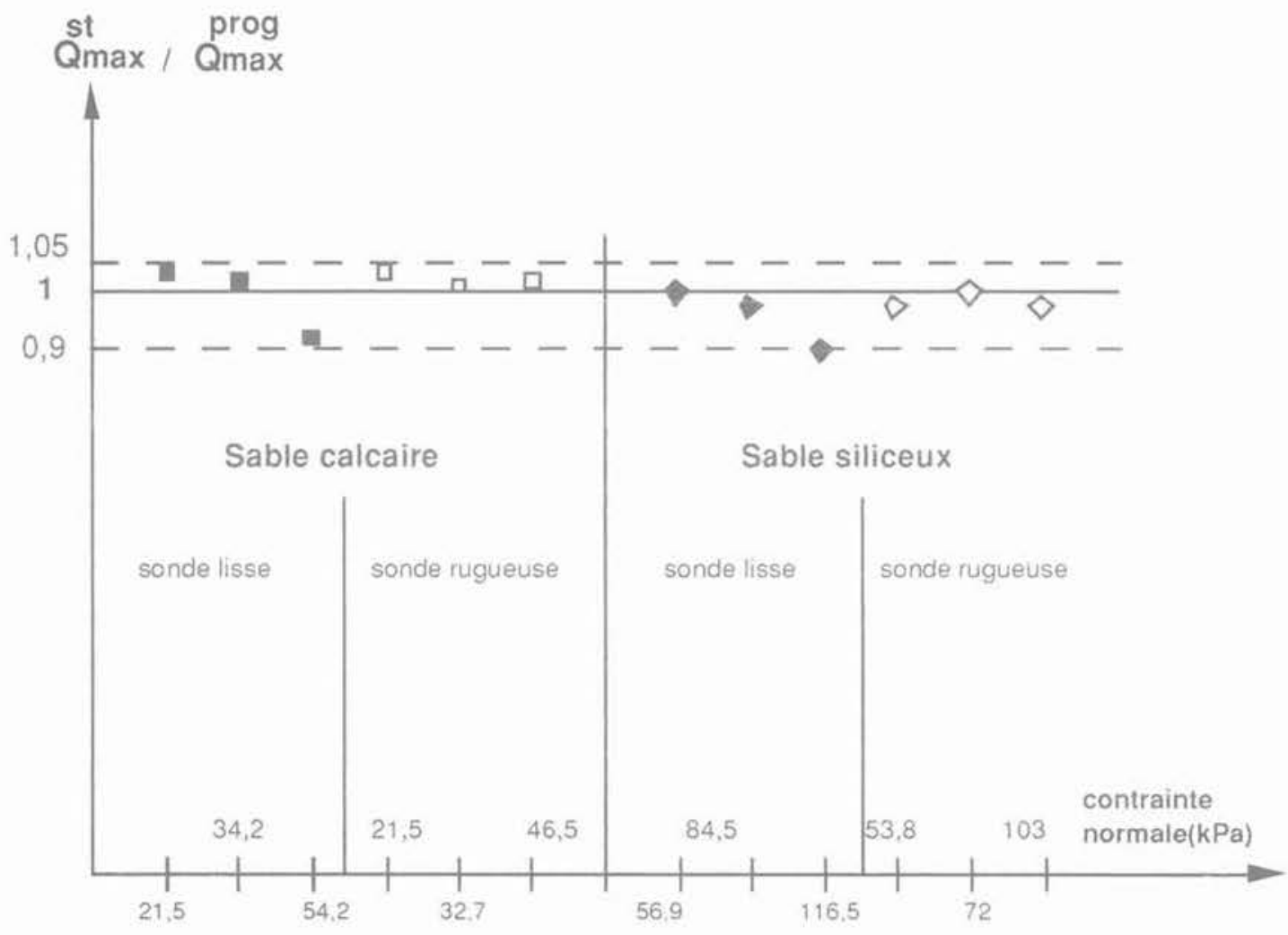

Fig. 14. - Rapport de l'effort limite déterminé durant un arrachement par paliers sur l'effort maximal atteint pendant l'arrachement progressif selon le type de sable et la rugosité de la sonde mini-pressiométrique $\left(\sigma_{V}=50 \mathrm{kPa}\right)$. Fig. 14. - Ratio of the limit static effort on the limit progressive effort for different roughtness of the probe and different kind of sands $\left(\sigma_{v}=50 \mathrm{kPg}\right)$

Enfin l'utilisation d'essais d'arrachement par paliers permet la définition d'une charge de fluage correspondant à l'apparition de déplacements importants.

\section{BIBLIOGRAPHIE}

(1) ABDERRAHIM A. (1991), Utilisation d'un minipressiomètre pour la mesure directe du frotte. ment à l'interface sol pulvérulent-inclusion. Doctorat de PINPL, np. 197.

(2) BERTHIER J. (1970), Essai statique de fonda. tions profondes. Projet du mode opératoire de LCPC, avril 1970.

(3) BOULON M., FORAY P., PLAYTAS C. (1986), Comportement dinterface et prévision du frottement latéral le long des pieux et tirants d'ancrage. Revue Française de Géotechnique, n०35, (1986), pp. 31-48.

(4) DAVIS A.G., PHILIPPONNAT G. (1978), Essais des pieux en vraie grandeur dans les milieux pulvérulents. Annales de l'Institut Technique du Bâtiment et des Travaux Publics, $\mathrm{n}^{\circ} 363,1978$, pp. 74-83.

(5) FAHEY M. (1986), Expansion of a thick cylinder of sand: a laboratory simulation of the pressuremeter test. Geotechnique vol. 36, n 3 , 1986, pp. 397-424.
(6) JEWELL R.J., FAHEY M. WROTH C.P. (1980), Laboratory studies of the pressuremeter test in sand. Geotechnique vol. 30, n 4, 1980, pp. 507-531.

(7) LE TIRANT P. et al. (1989), Frottement des pieux dans les sables carbonatés. Revue Française de Géotechnique, $n^{\circ} 49$, pp. 51-65.

(8) Ouvrage collectif (1991), Recommandations CLOUTERRE (1991), Presses ENPC Paris, pp. 159-183.

(9) PHILIPPONNAT G. (1986), Le phicomètre: essai de cisaillement direct dans le sol. Revue Française de Géotechnique, $n^{\circ} 35$, pp. 49-65.

(10) SCHLOSSER F., GUILLOUX (1981), Le frottement dans le renforcement des sols. Revue Française de Géotechnique, $n^{\circ} 16$, pp. 65-77.

(11) SCHLOSSER F, et al. (1979), Le renforcement sol-armature dans les ouvrages en terre armée. C. R. Coll. Int. Renforcement des sols, ENPC, Paris, pp. 151-156.

(12) YOSHIMI Y., KISHIDA T. (1981), A ring torsion apparatus for evaluating friction between soil and metal surfaces. Geotechnical Testing Journal GTDODJ, $n^{\circ} 4$, pp. 145-152.

(13) WERNICK (1978), Stresses and strains of the surface of anchors. Revue Française de Géotechnique, $n^{\circ} 3$, pp. 65-79. 